\title{
APROBACIÓN Y CREACIÓN DE TÍTULOS Y GRADOS EN LA PONTIFICIA UNIVERSIDAD CATÓLICA DE CHILE
}

Bernardo Domínguez* 


\section{RESUMEN}

En este trabajo se describen los procedimientos de creación y aprobación de los grados académicos y los títulos profesionales que otorga la Pontificia Universidad Católica de Chile.

Se señala que es el Consejo Superior de la Universidad quien tiene la atribución de creación de estos grados y títulos, y que para hacerlo, ha establecido procedimientos de evaluación y análisis con la participación de la Dirección Superior de la Universidad: Vicerrectoría Académica, académicos de sus Facultades (directivos y académicos) y de la comunidad externa a la Universidad (especialistas y pares evaluadores nacionales o extranjeros).

Estos procedimientos se vienen aplicando con rigurosidad desde hace más de veinte años en la Universidad, lo que ha permitido velar por un alto nivel de calidad de los programas de estudios ofrecidos, como también que su realización sea factible.

\section{ABSTRACT}

This paper describes the procedures used to create and adopt the academic degrees granted by the Pontificia Universidad Católica de Chile.

The paper explains that the University's Higher Council is responsible for instituting academic degrees and, to do so, it has established assessment and analysis procedures. Several of the University's higher academic units are involved in this process: the Office of the Academic Vice-President, academicians from the different schools, and external specialists and national and international peers from outside the university who perform assessments.

The rigorous implementation of these procedures for over twenty years has made it possible to ensure a high degree of quality in the study programs offered. It has also made their implementation feasible. 


\section{APROBACIÓN Y CREACIÓN DE TÍTULOS Y GRADOS EN LA PONTIFICIA UNIVERSIDAD CATÓLICA DE CHILE}

\section{INTRODUCCIÓN}

En la Pontificia Universidad Católica de Chile existe en la actualidad una gran variedad de programas de estudios que conducen a los distintos grados académicos, títulos profesionales, diplomas y menciones de especialización, así como certificados académicos o minores que se otorgan a los alumnos.

De acuerdo a lo establecido por los Estatutos Generales de la Universidad, corresponde al Consejo Superior, máximo organismo directivo colegiado, la atribución de crear, modificar o suprimir los títulos profesionales y grados académicos de la Universidad, acciones que se concretan a través de los correspondientes Decretos de Rectoría.

Para que el Consejo Superior pueda llevar a cabo de la mejor forma posible la creación de estos títulos y grados, se han establecido en la Universidad distintos procedimientos de evaluación y análisis en los que participan tanto las Facultades de la Universidad, a través de sus académicos, directivos y consejos académicos, como también la Dirección Superior de la Universidad, a través de sus Vicerrectorías Académica y Económica, y la comunidad externa a la Universidad, a través de especialistas, profesionales de prestigio o pares evaluadores académicos externos.

Estos procedimientos, establecidos inicialmente en 1978, se han ido perfeccionando y completando, de modo de asegurar el más alto nivel posible para los programas de estudios ofrecidos, como también que éstos sean factibles y permanentes en el tiempo. 


\section{CARACTERÍSTICAS DE LOS GRADOS ACADÉMICOS, TÍTULOS PROFESIONALES Y DIPLOMAS DE ESPECIALIDAD, ASÍ COMO CERTIFICADOS ACADÉMICOS OFRECIDOS POR LA UNIVERSIDAD.}

El nivel y forma de participación de los académicos, especialistas y directivos de la Universidad al analizar y aprobar nuevos programas de estudios, dependen del tipo de grado o título que ellos otorguen, como también si en dichos programas intervienen una o varias disciplinas (programas interdisciplinarios).

Los grados académicos que la Universidad ofrece actualmente son los de Bachiller, Licenciado, Magíster y Doctor.

El grado académico de Bachiller es el primer grado que otorga la Universidad. Está constituido por cursos de ciencias fundantes, de ciencias disciplinarias y cursos electivos, con una valoración total de 200 créditos académicos (cuatro semestres académicos de estudio en jornada completa). En la mayoría de los casos, los programas de estudio correspondientes a este grado son interdisciplinarios.

El grado académico de Licenciado se otorga después de aproximadamente cuatro o más años de estudios universitarios en jornada completa, valorados entre 400 y 500 créditos académicos. Este grado corresponde a la culminación de los estudios universitarios de pregrado.

En nuestra Universidad se aprobó recientemente que todos los programas de estudio correspondientes a las Licenciaturas, deben incluir en ellos el grado de Bachiller, a obtener durante el transcurso de dichos programas y previo al grado de Licenciado.

El grado académico de Magíster corresponde al grado inicial que se obtiene después de proseguir estudios universitarios de postgrado. Los programas correspondientes a este grado son en general monodisciplinarios y tienen una duración aproximada de tres a cuatro semestres académicos (150 a 200 créditos académicos adicionales al grado de Licenciado).

El grado académico de Doctor es el más alto grado que otorga la Universidad, y se obtiene después de realizar estudios avanzados e 
investigación al más alto nivel, con una duración aproximada entre seis y ocho semestres académicos (350 a 400 créditos), después de haber obtenido una Licenciatura.

Los programas de estudios del Doctorado pueden contener actividades desarrolladas en los programas de Magíster correspondientes.

Los títulos profesionales son otorgados por la Universidad después de realizar estudios que capaciten y habiliten a sus egresados para ejercer profesiones u oficios especializados. La mayoría de ellos tiene una duración de cinco o más años de estudio de dedicación jornada completa. Todos los títulos que otorga nuestra Universidad requieren de la obtención previa del grado académico de Licenciado.

Adicionalmente a los títulos y grados, la Universidad contempla en su organización académica la entrega de diplomas de especialidad, memorias de especialización y certificados académicos o minores.

En general, estos diplomas y certificados están insertos en los programas de estudios de algunos títulos y grados, y certifican la aprobación de un conjunto coherente de cursos en torno a una cierta área específica, con una valoración entre 50 y 100 créditos académicos.

\section{PROCEDIMIENTOS DE CREACIÓN DE NUEVOS GRADOS ACADÉMICOS, TÍTULOS PROFESIONALES Y CERTIFICADOS ACADÉMICOS.}

3.1 ¿Quiénes participan en la creación de nuevos programas de estudio?

En la elaboración, evaluación y aprobación de un nuevo proyecto de título o grado académico existen distintas instancias, en las que participan académicos y directivos de las Unidades Académicas y Facultades, especialistas y profesionales de alto nivel en las materias correspondientes (tanto nacionales como extranjeros) y directivos académicos y administrativos de la Dirección Superior de la Universidad. 


\section{2 ¿Cómo surgen las iniciativas?}

La creación de una carrera y/o grado académico puede surgir como iniciativa de una Facultad o Unidad Académica, ya sea a través de un profesor, de un grupo de profesores o de su Dirección. Por otra parte, también la iniciativa puede provenir de la Dirección Superior de la Universidad, a través de alguna de las Direcciones de su Vicerrectoría Académica: la Dirección General de Pregrado, la Dirección General de Investigación y Postgrado (DIPUC) o la Dirección General de Desarrollo Académico. Generalmente cuando se trata de un grado académico con programa de estudio interdisciplinario, la iniciativa es de la Dirección Superior.

\section{3 ¿Quién prepara y aprueba los anteproyectos?}

Si la idea del proyecto surge en alguna Unidad Académica, su elaboración y aprobación le corresponde al principal organismo colegiado que tenga la Facultad: el Consejo de Facultad. Para esta labor, el Consejo puede designar las comisiones que estime conveniente, incluyendo tanto académicos como especialistas externos.

En el caso de grados académicos con programas interdisciplinarios, corresponde a la Vicerrectoría Académica la elaboración del anteproyecto para desarrollarlo. Para estos efectos, ésta crea una comisión especial integrada tanto por académicos de las Facultades pertinentes como por representantes de la Vicerrectoría Académica. De todas formas, las Facultades que intervienen deben analizar estos programas con el objeto de darles su respaldo y aprobación.

\section{4 ¿Qué aspectos debe contener un nuevo proyecto de grado o título?}

Los anteproyectos de nuevos programas de estudios deben ser preparados y diseñados desde una triple perspectiva:

- Fundamentación y justificación académica.

- Factibilidad de implementarlo en atención a los recursos humanos (personal académico, técnico y administrativo) y a los recursos 
materiales (infraestructura, equipamiento, laboratorios, bibliotecas).

- Factibilidad económica y pertinencia de su creación, tanto desde la perspectiva interna de la Universidad, como de la conveniencia social para el país.

Por ello, estos anteproyectos deben incluir un conjunto de antecedentes académicos, de infraestructura y de recursos humanos y económicos.

Como ejemplo, se presentan en los Anexos I y II las pautas académicas y económicas que usa actualmente la Universidad, y que están a disposición de las Facultades para la elaboración de los anteproyectos.

\section{5 ¿Quién y cómo se evalúa un anteproyecto?}

La Vicerrectoría Académica de la Universidad es la responsable del análisis y evaluación de los nuevos proyectos de títulos profesionales y grados académicos. Por ello, los anteproyectos, después de haber sido aprobados por los Consejos de Facultad respectivos, son enviados a dicha Vicerrectoría para su evaluación.

En el caso de un anteproyecto para la creación de un programa de postgrado, la Vicerrectoría Académica lo somete a un análisis por parte de DIPUC, que revisa si el proyecto contiene todos los antecedentes necesarios para su evaluación académica y si se ajusta al Reglamento de Postgrado de la Universidad y a los estándares de calidad que se manejan en ella y también los que tiene la Comisión Nacional de Acreditación de Postgrados (CONAP).

Realizadas todas las observaciones del caso por parte de la Vicerrectoría Académica, se inicia un proceso interactivo de correcciónrevisión del documento del anteproyecto, hasta que éste se ajusta a los requerimientos de DIPUC. En esta etapa se produce un diálogo entre los coordinadores del anteproyecto para lograr un documento o proyecto en que estén de acuerdo tanto la Dirección Superior como la Facultad correspondiente. 
Dicho documento -aprobado por DIPUC- es enviado a evaluación por dos pares académicos externos a la Universidad, donde al menos uno debe ser del extranjero. Recibidas las observaciones de los expertos, éstas se hacen llegar a los coordinadores del proyecto, quienes deben responderlas y, cuando proceda, modificar el documento originalmente aprobado.

En forma paralela a la evaluación externa, el proyecto es enviado a la Dirección de Finanzas de la Vicerrectoría de Asuntos Económicos y Administrativos, donde se analiza su factibilidad económica.

Una vez que DIPUC recibe el documento modificado de parte de la Unidad Académica, (siguiendo las observaciones de los evaluadores externos), así como también el informe económico de la Dirección de Finanzas, el proyecto pasa a manos del Vicerrector Académico para su eventual aprobación como proyecto definitivo, a ser presentado al Consejo Superior.

Los anteproyectos correspondientes a nuevos títulos profesionales se estudian y analizan en forma similar, pero en este caso es un proceso liderado y coordinado por la Dirección General de Pregrado (Dirección de Docencia). Ésta revisa que el anteproyecto contenga todos los antecedentes necesarios y lo entrega para el análisis de una Comisión Académica ad-hoc, nombrada por el Vicerrector, en la que participan representantes de la Vicerrectoría Académica, de la Facultad pertinente y uno o más especialistas expertos en el tema, no vinculados al anteproyecto. Una forma alternativa consiste en mandarlo a evaluadores externos a la Universidad, seleccionados por su experiencia, tanto académica como profesional.

La Comisión Académica emite un informe que, en caso necesario, se hace llegar a la Facultad respectiva, a fin de incorporar eventuales modificaciones solicitadas o justificar su no procedencia.

Estos anteproyectos, al igual que los de postgrado, se envían a la Dirección de Finanzas de la Vicerrectoría de Asuntos Económicos y Administrativos, para que ésta realice una evaluación económica y emita el informe correspondiente. 
Basándose en estos informes el Vicerrector Académico aprueba un proyecto definitivo para ser presentado a la instancia final del Consejo Superior.

\subsection{Tramitación final y aprobación definitiva del Consejo Superior de la Universidad}

El Vicerrector Académico envía el proyecto y demás antecedentes (evaluación de pares, observaciones, informe económico, etc.) al Secretario General, quien convoca a la Comisión de Títulos y Grados del Consejo Superior, "encargada de asesorar e informar al Consejo sobre la creación, modificación o supresión de cualquier título profesional o grado académico", para que analice el proyecto.

En esta instancia participan los Decanos miembros permanentes de la referida comisión, el Secretario General, el Vicerrector Académico, el Director de DIPUC o de Pregrado, según sea el caso, el Decano que presenta el proyecto y el coordinador de éste. El proyecto puede ser aprobado, aprobado con modificaciones, o rechazado. Si es aprobado con modificaciones y éstas son de consideración, el proyecto deberá ser presentado en una segunda oportunidad ante esta Comisión. Si las modificaciones sugeridas son menores, es la Vicerrectoría Académica quien se responsabiliza de que se efectúen dichos cambios.

Una vez que el proyecto cumple con todas las exigencias de la Comisión de Títulos y Grados se envía, junto con todos los antecedentes acumulados, al Consejo Superior. En esta instancia se produce el análisis final y definitivo del proyecto.

Si el proyecto es aprobado por el Consejo Superior, se emite un Decreto de Rectoría que crea el título o grado y su programa respectivo, así como también una Resolución de la Vicerrectoría Académica que reglamenta su funcionamiento.

\section{ALGUNAS CONSIDERACIONES FINALES}

En esta Universidad, en los últimos años, la actividad de creación o modificación de carreras conducentes a título profesional y grados académicos ha sido intensa. 
En el nivel de postgrado la Universidad cuenta en la actualidad con 52 programas de Magíster (con sus distintas menciones) y 18 programas de Doctorado. En este año 2002, hay 7 programas de Magíster y dos de Doctorado en etapa de estudio y proceso de aprobación.

En el nivel de pregrado se aprobó en el año 2001, tras un largo y completo proceso de estudio, incorporar el grado de Bachiller a todas las carreras profesionales y los estudios de Licenciaturas. Esto ha obligado a revisar y reestructurar todos los planes de estudio, en un intenso trabajo conjunto de las Unidades Académicas y la Vicerrectoría Académica.

A pesar de lo dinámica y compleja que es esta labor, la Universidad estima que debe continuar realizándola con la mayor acuciosidad posible, ya que es de suma importancia para mantener el más alto nivel de calidad en sus actividades docentes y lograr que ellas se adecuen permanentemente a los requerimientos de la sociedad y del país.

En estas condiciones la Universidad debe utilizar todas las posibilidades que actualmente le da el sistema universitario, tanto nacional como internacional. Por esta razón la Universidad está impulsando a las Facultades para que, desde su creación, los nuevos programas de estudios se presenten tanto ante la Comisión Nacional de Acreditación (CONAP), como ante agencias acreditadoras internacionales para su estudio y evaluación, de modo de recibir su opinión y observaciones y lograr de esta manera que, desde sus inicios, estos nuevos programas se desarrollen de la mejor forma posible. 


\section{ANEXO I}

GUÍA PARA PRESENTACIÓN DE PROYECTOS QUE CREAN NUEVOS PROGRAMAS DE ESTUDIOS CON EL OBJETO DE REALIZAR SU EVALUACIÓN ACADÉMICA

Para que un anteproyecto pueda ser evaluado, desde un punto de vista académico, debe incluir los siguientes antecedentes:

1. Antecedentes y justificación para la creación del nuevo programa de estudios. Señalar cómo se insertaría este programa en los planes de desarrollo académico y en las líneas de investigación de la Unidad Académica correspondiente; por qué es recomendable crear el título profesional, el grado académico o la mención en nuestra Universidad y cuál sería la demanda por el programa y sus egresados, entre otros.

2. Objetivos generales y específicos del grado o título.

3. Habilidades que deberá desarrollar el alumno.

4. Currículum y plan de estudios. Incluir la nómina de cursos mínimos y optativos y la malla curricular.

5. Se debe adjuntar el programa específico incluyendo:

- Sigla propuesta para el curso

- Nombre del curso

- Créditos que otorgará

- Requisitos para tomar el curso

- Profesor encargado

- Módulos docentes

- Objetivos

- Contenidos

- Metodología

- Evaluación

- Bibliografía

6. Características de la tesis, taller final o seminario.

7. Requisitos de ingreso, requisitos de egreso y de graduación o titulación.

8. Nómina de profesores participantes. Ésta debe contener:

- Nombre del profesor

- Grado(s) académico(s) y/o título profesional

- Institución que otorgó grado(s) y título 
- Año de obtención de los mismos

- Categoría académica

- Tipo de jornada

- Tiempo de dedicación al programa de postgrado

- Proyectos de investigación y publicaciones en los últimos tres años.

9. Reglamento del Programa de Estudios. En caso de tratarse de un programa de postgrado, debe estructurarse en títulos y artículos, que deberán referirse a los siguientes aspectos:

- Definición del programa de postgrado

- Dirección del programa de postgrado

- Requisitos de ingreso

- Currículum

- Exigencias académicas

- Características de la tesis

- Requisitos de egreso y graduación

Este reglamento debe ser coherente con el Reglamento General de Estudios de Postgrado y el Reglamento del Alumno de Postgrado que rigen en nuestra Universidad. 


\section{ANEXO II}

PAUTA DE ANTECEDENTES A PRESENTAR PARA LA EVALUACIÓN ECONÓMICA DEL NUEVO PROGRAMA DE ESTUDIOS POR PARTE DE LA DIRECCIÓN DE FINANZAS DE LA UNIVERSIDAD

\begin{tabular}{|c|c|}
\hline Parámetro de ingresos & Parámetros de egresos \\
\hline Ingresos directos & \begin{tabular}{|l|} 
Profesores/curso \\
(\$/profesor/curso/mes)
\end{tabular} \\
\hline Arancel total programa $(\$)$ & Ayudantes/curso \\
\hline Otros ingresos directos $1(\%)$ & (\$/ayudante/curso/mes) \\
\hline & Sueldo administrativos \\
\hline Ingresos indirectos & Dirección del programa (\$/mes) \\
\hline & Secretaría del programa (\$/mes) \\
\hline Otros ingresos & \\
\hline Otros ingresos 1 & Gtos. administrativos (\% matrícula) \\
\hline Crec. otros ingresos 1 por periodo & \\
\hline Otros ingresos 2 & Publicidad \\
\hline Crec. otros ingresos 2 por periodo & Publicidad al inicio del programa \\
\hline Otros ingresos 3 & Publicidad periódica \\
\hline Crec. otros ingresos 3 por periodo & \\
\hline & Arriendo salas (\$/módulo) \\
\hline & Becas matrícula (alumnos/periodo) \\
\hline & Otros egresos \\
\hline & Egresos directos \\
\hline & Otros egresos indirectos $1(\$)$ \\
\hline & Crec. otros egresos 1 por periodo \\
\hline & Inversión \\
\hline & Inversión inicial (\$) \\
\hline & Valor residual de la inversión \\
\hline
\end{tabular}




\section{Parámetros generales}

\begin{tabular}{|l|l|}
\hline Periodicidad del programa & Semestral \\
\hline Duración del programa ( ${ }^{o}$ periodos) & \\
\hline Promociones /año & \\
\hline Periodo de evaluación & \\
\hline
\end{tabular}

\begin{tabular}{|l|l|}
\hline$N^{\circ}$ alumnos por promoción & \\
\hline Tasa deserción de alumnos por periodo (\%) & \\
\hline Aumento alumnos matriculados por prom. & \\
\hline
\end{tabular}

\begin{tabular}{|l|l|}
\hline Participación central & \\
\hline Tasa de descuento anual & \\
\hline
\end{tabular}

\begin{tabular}{|c|l|l|}
\cline { 2 - 3 } \multicolumn{1}{c|}{ Malla curricular } & No de cursos & No de módulos \\
Cursos & periodo \\
\hline Periodo & & \\
\hline 1 & & \\
\hline 2 & & \\
\hline 3 & & \\
\hline 4 & & \\
\hline 5 & & \\
\hline 6 & & \\
\hline 7 & & \\
\hline 8 & & \\
\hline 9 & & \\
\hline 10 & & \\
\hline
\end{tabular}




\section{FLUJO}

\begin{tabular}{|l|c|c|c|c|c|c|c|c|c|c|c|}
\hline & \multicolumn{10}{|c|}{ Período } \\
\cline { 2 - 13 } & 0 & 1 & 2 & 3 & 4 & 5 & 6 & 7 & 8 & 9 & 10 \\
\hline INVERSIÓN INICIAL & & & & & & & & & & & \\
\hline Valor residual inversión & & & & & & & & & & & \\
\hline
\end{tabular}

INGRESOS

\begin{tabular}{|l|l|l|l|l|l|l|l|l|l|l|l|}
\hline Matrícula & & & & & & & & & & & \\
\hline Otros ingresos directos & & & & & & & & & & & \\
\hline Otros ingresos indirectos (1) & & & & & & & & & & & \\
\hline Otros ingresos indirectos (2) & & & & & & & & & & & \\
\hline Otros ingresos indirectos (3) & & & & & & & & & & & \\
\hline Total ingresos & & & & & & & & & & & \\
\hline
\end{tabular}

\section{EGRESOS}

\begin{tabular}{|l|l|l|l|l|l|l|l|l|l|l|l|}
\hline Rem. profesores & & & & & & & & & & & \\
\hline Rem. ayudantes & & & & & & & & & & & \\
\hline Dirección del programa & & & & & & & & & & & \\
\hline Secretaría del programa & & & & & & & & & & & \\
\hline Gastos administrativos & & & & & & & & & & & \\
\hline Publicidad (inversión) & & & & & & & & & & & \\
\hline Publicidad periódica & & & & & & & & & & & \\
\hline Arriendo salas & & & & & & & & & & & \\
\hline Becas matrículas & & & & & & & & & & & \\
\hline Otros egresos directos & & & & & & & & & & & \\
\hline Otros egresos indirectos & & & & & & & & & & & \\
\hline Participación central & & & & & & & & & & & \\
\hline Total egresos & & & & & & & & & & & \\
\hline
\end{tabular}

Ingresos - Egresos

\section{VAN}

